\title{
Precise predictions for electroweakino-pair production in association with a jet at the LHC
}

\author{
Julien Baglio, ${ }^{a, b, c}$ Barbara Jäger $^{a}$ and Matthias Kesenheimer ${ }^{a}$ \\ ${ }^{a}$ Institute for Theoretical Physics, University of Tübingen, \\ Auf der Morgenstelle 14, 72076 Tübingen, Germany \\ ${ }^{b}$ Institute for Advanced Study, Durham University, \\ Cosin's Hall, Palace Green, Durham DH1 3RL, United Kingdom \\ ${ }^{c}$ Institute for Particle Physics Phenomenology, Department of Physics, Durham University, \\ South Road, Durham DH1 3LE, United Kingdom \\ E-mail: julien.baglio@uni-tuebingen.de, \\ barbara.jaeger@itp.uni-tuebingen.de, \\ matthias.kesenheimer@uni-tuebingen.de
}

ABSTRACT: We present the full NLO SUSY-QCD corrections to the pair production of neutralinos and charginos at the LHC in association with a jet and their matching to partonshower programs in the framework of the POWHEG-BOX package. The code we have developed provides a SUSY Les Houches Accord interface for setting electroweak and supersymmetric input parameters. Decays of the neutralinos and charginos and parton-shower effects can be simulated with multi-purpose programs such as PYTHIA. The capabilities of the code are illustrated by phenomenological results for a parameter point in the framework of pMSSM10, compatible with present experimental limits on supersymmetry. We find that NLO-QCD corrections as well as parton-shower effects are of primary importance for the appropriate description of jet distributions.

KeYwords: NLO Computations, Supersymmetry Phenomenology

ArXiv EPrint: 1711.00730 


\section{Contents}

1 Introduction 1

2 Framework of the calculation 3

2.1 Virtual corrections 4

$\begin{array}{lll}2.2 & \text { Real emission corrections } & 6\end{array}$

3 Phenomenological results $\quad 9$

$\begin{array}{ll}3.1 \text { Choice of the spectrum and input parameters } & 10\end{array}$

$\begin{array}{lll}3.2 & \text { Cross sections and distributions at the LHC } & 10\end{array}$

4 Conclusions $\quad 15$

\section{Introduction}

After a very successful Run I of the CERN Large Hadron Collider (LHC) at energies of 7 and $8 \mathrm{TeV}$ in which, notably, the ATLAS and CMS collaborations discovered a particle with properties compatible with a Standard Model (SM) Higgs boson [1, 2], LHC Run II at $13 \mathrm{TeV}$ started in 2015. This unprecedented energy allows to probe an uncharted territory that may conceal new elementary particles. Even though the observation of a Higgs boson has advanced our understanding of the electroweak symmetry breaking mechanism, important questions remain open that are pointing towards the need for physics beyond the SM (BSM). In particular, astrophysical and cosmological observations strongly imply the existence of Dark Matter (DM), a new type of matter that interacts only weakly with SM particles. The SM does not contain any elementary particles that are suitable candidates for DM. Amongst the different BSM scenarios on the market, supersymmetry (SUSY) offers a very promising avenue to account for DM (see, e.g., ref. [3] for a recent review). SUSY features new particles that differ from their SM counterparts by their spin and acquire large masses by the mechanism of SUSY breaking. In the minimal supersymmetric extension of the SM (the MSSM), the conservation of a discrete symmetry called R-parity ensures that the lightest SUSY particle (LSP) is stable. In many SUSY scenarios this particle is the lightest neutralino, a mixture of the superpartners of the Higgs, the photon, and the $Z$ bosons. Being stable and electrically neutral, it provides an excellent candidate for fermionic DM. In the following, we will refer to neutralinos and charginos generically as electroweakinos or simply weakinos.

A very clean signature of DM at the LHC would be provided by a significant amount of missing transverse energy in association with a tagging jet or lepton, so-called mono-jet or mono-lepton signatures. These can be produced by LSP pairs in two ways: either by the production of heavier SUSY particles that decay into LSPs and additional leptons or 
jets; or by the direct production of a pair of LSPs in association with a jet. Weakino-pair production is a pure electroweak (EW) process at lowest order. As a consequence, the associated production cross sections are small, and the respective exclusion limits on the MSSM parameters obtained by ATLAS and CMS are less severe than those obtained by the QCD mediated squark and gluino production processes with significantly larger cross sections, see e.g. refs. [4-7] at $8 \mathrm{TeV}$ and refs. [8-10] at $13 \mathrm{TeV}$. Thus, direct production of weakino pairs plus one identified jet resulting in mono-jet signatures is of particular phenomenological relevance.

The direct production of weakino pairs without an additional jet at the lowest order has been studied for decades. The first calculation of the next-to-leading-order (NLO) SUSYQCD corrections to the total cross section at hadron colliders was presented in ref. [11]. The public computer program PROSPINO [12] contains the NLO SUSY-QCD corrections for the total cross sections. Resummation effects, in particular for the transverse momentum distributions, were provided in ref. [13]. That work showed that leading-order (LO) calculations are not appropriate for predicting transverse momentum spectra. Threshold corrections were provided in refs. $[14,15]$ and the combination of the latter with transversemomentum resummation effects was provided in ref. [16] and implemented in the public package RESUMMINO [17]. As far as EW corrections are concerned, they were calculated in ref. [18] for the associated production of a chargino and a neutralino at the LHC and found to be moderate for representative parameter points. We provided an interface of the NLO SUSY-QCD calculation for weakino-pair production to parton shower programs [19] via the POWHEG matching procedure [20, 21], allowing for simulations including parton-shower, underlying-event, and multi-parton interaction effects using Monte-Carlo programs such as PYTHIA [22].

Predictions for weakino-pair production in association with an additional jet are less advanced. The LO differential cross sections for all types of weakino pairs plus an extra hard jet were available in ref. [23] (and references therein), while the NLO SUSY-QCD corrections for the neutralino-pair production mode in association with a hard jet were calculated in ref. [24]. The latter calculation, however, does not generically account for the complicated structure of the on-shell resonances that may appear in the real-emission contributions of such processes. Moreover, no dedicated interface of an NLO-QCD calculation to parton-shower programs exists yet.

In this paper we provide that missing piece building on experience gained in the implementation of weakino-pair production at the LHC [19]. We present a code package for all weakino-pair production processes (i.e. neutralino-neutralino, neutralino-chargino and chargino-chargino) in association with an identified jet at the LHC, including the full NLO SUSY-QCD corrections to cross sections and differential distributions within arbitrary experimental selection cuts, matched to parton shower programs via the POWHEG method. We use the framework of the POWHEG-BOX [25], a public repository for the simulation of scattering processes at hadron colliders at NLO-QCD accuracy matched with parton shower programs. We provide, for the first time, the subtraction of the on-shell resonance structure of the real-emission corrections to a process with three particles in the final state at the lowest order. We find that the SUSY-QCD corrections modify the differential cross 
a)

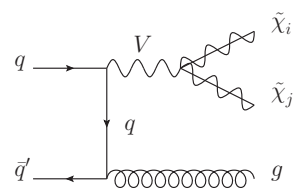

b)
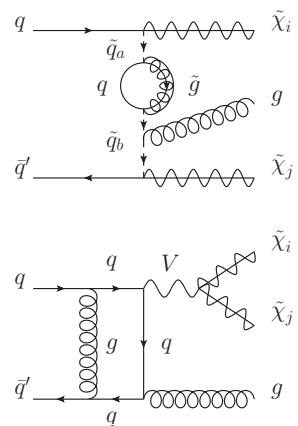
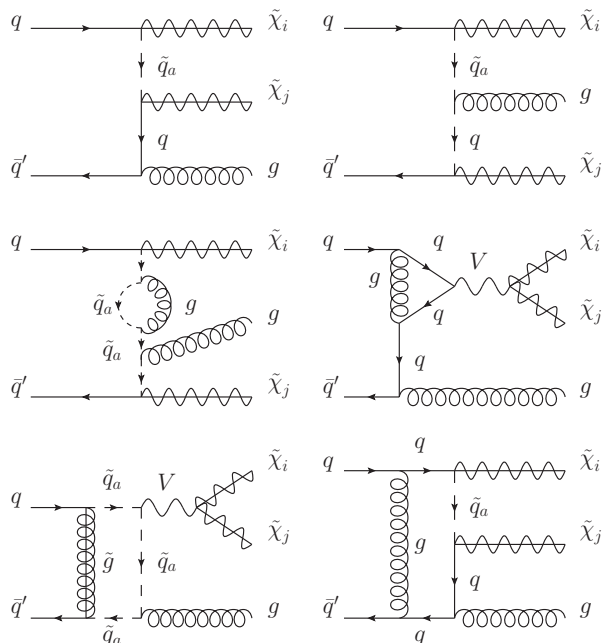
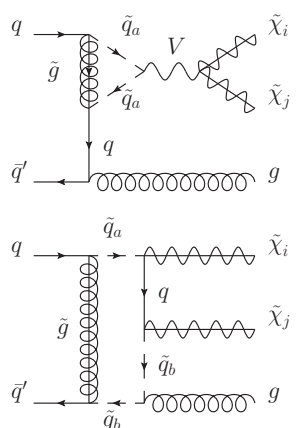

Figure 1. Representative (a) tree-level and (b) one-loop diagrams for the production of a pair of weakinos, $\tilde{\chi}_{i} \tilde{\chi}_{j}$, with a jet. Depending on the types $i, j$ of the produced weakinos, $V$ stands for $W^{ \pm} / Z / \gamma$, and $a, b=1,2$.

sections by up to $15 \%$ in certain phase-space regions at the LHC, in a way that cannot be accounted for with a constant $K$-factor.

In the following section, we briefly describe the technical aspects of our calculation that are specific to the implementation of weakino-pair production processes in the context of the POWHEG-BOX, in particular the subtraction of the on-shell resonances. In section 3 we provide representative numerical results using a parameter point in the framework of the pMSSM10 [26], compatible with present experimental limits on SUSY. This illustrates the importance of NLO SUSY-QCD and parton-shower effects in the correct description of the jet activity in DM mono-jet searches at the LHC. Our conclusions are given in section 4.

\section{Framework of the calculation}

Our calculation of the production of a pair of weakinos in association with a jet builds on the experience we have gained in our previous implementation of weakino-pair production processes in the framework of the POWHEG-BOX [19]. In this section, we present the specific aspects of our implementation and, in particular, extensions with respect to the calculation of weakino-pair production.

At the lowest order the production of a pair of weakinos in association with a jet proceeds via three parton-level channels: $q \bar{q}^{\prime} \rightarrow \tilde{\chi}_{i} \tilde{\chi}_{j} g, q g \rightarrow \tilde{\chi}_{i} \tilde{\chi}_{j} q$, and $\bar{q} g \rightarrow \tilde{\chi}_{i} \tilde{\chi}_{j} \bar{q}$, where $\tilde{\chi}_{i}$ stands for either a neutralino $\tilde{\chi}_{i}^{0}(i=1 \cdots 4)$, or a chargino $\tilde{\chi}_{i}=\tilde{\chi}_{i}^{ \pm}(i=1,2)$. Some representative diagrams for the $q \bar{q}^{\prime}$-induced partonic channels are depicted in figure $1 \mathrm{a}$ ). We assume four massless active quark flavors in the running of the strong coupling constant and take finite bottom-quark and top-quark mass effects emerging in loop diagrams into account in the calculation. The Cabibbo-Kobayashi-Maskawa matrix is taken diagonal and we also use four-flavor-scheme parton distribution functions with no bottom-quark initial states. 
In the gluon-induced channels $q(\bar{q}) g \rightarrow \tilde{\chi}_{i} \tilde{\chi}_{j} q(\bar{q})$, single resonances can already occur at the lowest order when an intermediate squark happens to be on-shell in an $s$-channel diagram. These resonance effects are regulated by using the complex-mass scheme [27]. We replace the mass of the squark, $m_{\tilde{q}_{k}}$, with a complex mass $\mu_{\tilde{q}_{k}}$ such that $\mu_{\tilde{q}_{k}}^{2}=m_{\tilde{q}_{k}}^{2}-$ $i \Gamma_{\tilde{q}_{k}} m_{\tilde{q}_{k}}$, where $\Gamma_{\tilde{q}_{k}}$ denotes the physical decay width of the squark. We stress that this procedure is gauge invariant.

\subsection{Virtual corrections}

The virtual corrections to the three parton-level processes $q \bar{q}^{\prime} \rightarrow \tilde{\chi}_{i} \tilde{\chi}_{j} g, q g \rightarrow \tilde{\chi}_{i} \tilde{\chi}_{j} q$, and $\bar{q} g \rightarrow \tilde{\chi}_{i} \tilde{\chi}_{j} \bar{q}$ are classified as self-energy, triangle, box, and pentagons corrections, with gluon, gluino, quark, or squark exchange, see figure $1 \mathrm{~b}$ ) for some representative diagrams. In our calculation, diagrams containing a neutral Higgs boson coupling to top-quark and squark loops and decaying to a pair of weakinos are included. Resonance effects of these Higgs particles in $s$-channel diagrams are regulated by a finite Higgs width. We have used FeynArts 3.9 [28] to generate the virtual diagrams and FormCalc 9.4 [29] to calculate, in the Feynman-'t Hooft gauge, the amplitudes using the MSSM-CT model file of ref. [30]. In order to regularize single resonances that we encounter already at the Born level, the complex-mass scheme [27] for squark and gluino masses is used. Scalar and tensor one-loop integrals are numerically evaluated with the computer package COLLIER-1.0 [31].

For the regularization of ultraviolet (UV) divergences we follow the procedure of our previous calculation and use the dimensional regularization scheme (DREG), the standard procedure of the POWHEG-BOX. The entire calculation is done in $D=4-2 \varepsilon$ dimensions, which is known to break supersymmetry at the level of the gauge interactions by introducing a mismatch in the $(D-2)$ transverse degrees of freedom of the gauge bosons and the two degrees of freedom of the gauginos. In particular, this means that the quark-squark-weakino Yukawa coupling $\hat{g}$ and the associated $\mathrm{SU}(2)$ gauge coupling $g$ are no longer equal to all orders in the perturbative expansion. To restore this symmetry, we introduce a finite SUSY restoring counter-term at NLO in the strong coupling $\alpha_{s}[11,32,33]$,

$$
\hat{g}=g\left(1-\frac{\alpha_{s}}{6 \pi}\right) \text {. }
$$

The expansion in $\alpha_{s}$ is done consistently to retain only the $\mathcal{O}\left(\alpha_{s}\right)$ term that is induced by this finite SUSY restoring counter-term in the amplitude squared.

In order to cancel the UV divergences we perform a renormalization procedure and calculate the suitable counter-terms necessary to define finite physical input parameters. We use the on-shell scheme for the renormalization of the wave functions of external colored particles (quarks, gluons), as well as for the squark, gluino, and top masses emerging in internal propagators. The strong coupling constant $\alpha_{s}$, however, is renormalized in the $\overline{\mathrm{MS}}$ scheme. In particular, the renormalization constant of the strong coupling constant, $\delta Z_{g_{s}}$, is calculated using the gluon field renormalization constant $\delta Z_{g g}$ provided by FormCalc (note that $\delta Z_{g g}$ is in principle a gauge-dependent renormalization constant and is here calculated in the Feynman-'t Hooft gauge). We thus have

$$
\delta Z_{g_{s}}=-\left.\frac{3}{2} \delta Z_{g g}\right|_{\mathrm{div}}+\delta Z_{3 g}+\delta Z_{\mathrm{log}}
$$


with $\left.\delta Z_{g g}\right|_{\text {div }}$ being the divergent part of the gluon field renormalization constant,

$$
\left.Z_{g g}\right|_{\text {div }}=-\frac{3}{4 \pi} \alpha_{s} \Delta
$$

and

$$
\delta Z_{3 g}=-\frac{3}{2 \pi} \alpha_{s} \Delta
$$

in the MSSM, with $\Delta=1 / \varepsilon-\gamma_{E}+\ln 4 \pi$ in the $\overline{M S}$ scheme. The quantity $\gamma_{E}$ is the EulerMascheroni constant. The last term in eq. (2.2) is an additional finite shift to account for the decoupling of heavy particles in the running of the strong coupling constant $\alpha_{s}$. The decoupling of heavy particles has first been discussed in ref. [34] and can be understood as follows.

The renormalization counterterm for the strong coupling constant can be associated with the $\beta$-function, defined by the renormalization group equation of $\mathrm{QCD}$,

$$
\mu_{R}^{2} \frac{d}{d \mu_{R}^{2}} \alpha_{s}=\beta\left(\alpha_{s}\right)=-\alpha_{s}^{2} \sum_{n} \beta_{n} \alpha_{s}^{n},
$$

where $\mu_{R}$ is the renormalization scale. At the one-loop order we have (see, for example, ref. [35]) $\delta Z_{g_{s}}=-\alpha_{s} \Delta \beta_{0} / 2$. With eqs. (2.2)-(2.4), this means $\beta_{0}=3 / 4 \pi$, and all particles, including the heavy states, are contributing to the running of $\alpha_{s}$, leading to potentially large logarithms, if the scale $\mu_{R}$ is significantly different from the masses of the various heavy states of the theory. In the SM with only four active flavors contributing to the running of $\alpha_{s}$, we have $\beta_{0}^{\text {light }}=25 / 12 \pi$. Following ref. [35], the heavier states can be subtracted by rescaling $\alpha_{s}$ so that only the light four active quark flavors contribute to the running of $\alpha_{s}$ as in the SM, with the bottom and top quarks taken as massive, decoupled particles. This can be achieved in our calculation by adding the finite term $\delta Z_{\log }$ to the strong coupling constant counterterm, to subtract the logarithms that arise from the masses of squarks $\left(m_{\tilde{q}_{i}}\right)$, gluino $\left(m_{\tilde{g}}\right)$, top quark $\left(m_{t}\right)$ and bottom quark $\left(m_{b}\right)$,

$$
\delta Z_{\log }=-\frac{\alpha_{s}}{8 \pi}\left[2 \ln \frac{m_{\tilde{g}}^{2}}{\mu_{R}^{2}}+\frac{1}{6} \sum_{i=1}^{12} \ln \frac{m_{\tilde{q}_{i}}^{2}}{\mu_{R}^{2}}+\frac{2}{3} \ln \frac{m_{t}^{2}}{\mu_{R}^{2}}+\frac{2}{3} \ln \frac{m_{b}^{2}}{\mu_{R}^{2}}\right] .
$$

With this shift the one-loop running of $\alpha_{s}$ with four light degrees of freedom is recovered:

$$
\begin{aligned}
\mu_{R}^{2} \frac{d}{d \mu_{R}^{2}} \alpha_{s} & =-\alpha_{s}\left[\beta_{0}-\frac{1}{4 \pi}\left(-2-2-\frac{2}{3}-\frac{2}{3}\right)\right] \\
& =-\alpha_{s} \beta_{0}^{\text {light }} .
\end{aligned}
$$

Other fundamental parameters emerging in our calculation, such as the electroweak coupling constant, do not require renormalization at NLO in QCD. 


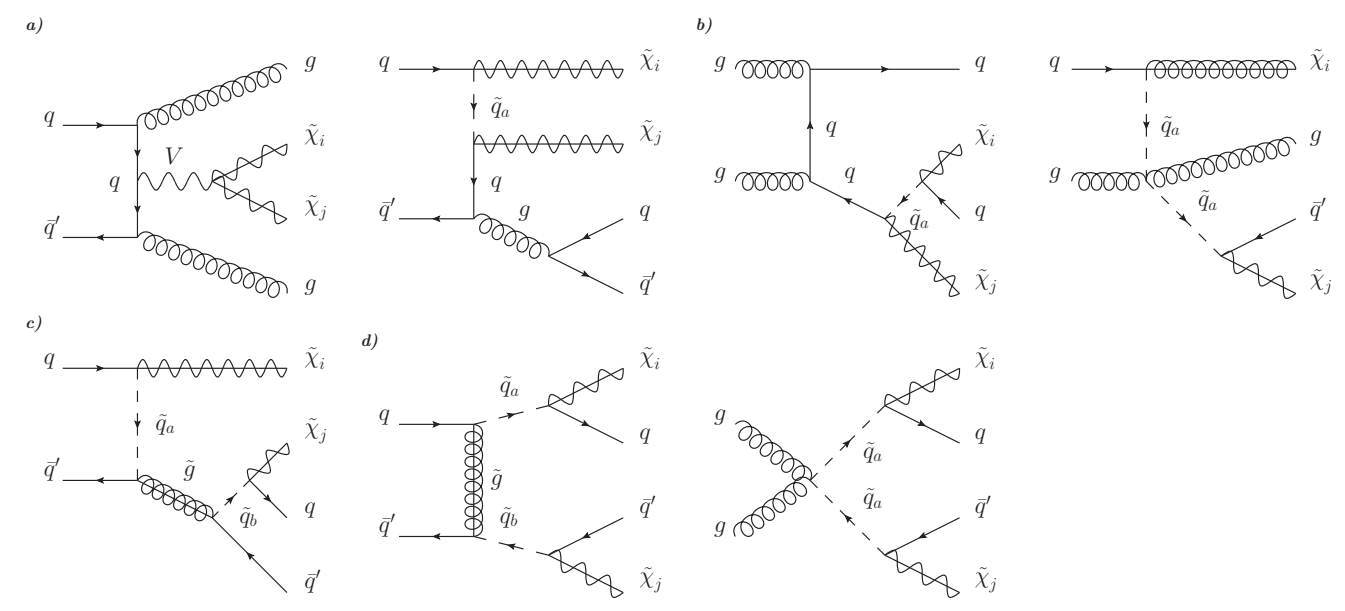

Figure 2. Representative (a) non-resonant, (b) squark singly-resonant, (c) gluino singly-resonant, and (d) squark doubly resonant real-emission diagrams for the production of a pair of weakinos with a jet.

\section{$2.2 \quad$ Real emission corrections}

The calculation of the real-emission corrections to weakino-pair production in association with a jet requires the evaluation of all partonic subprocesses contributing to the reaction $p p \rightarrow \tilde{\chi}_{i} \tilde{\chi}_{j} j j$. This includes quark-anti-quark annihilation processes with two gluons in the final state and all possible crossed modes, as well as the additional class of processes with two (anti-)quarks in the initial and in the final state. Matrix elements for all of these subprocesses can in principle be generated with the help of automated tools, such as the generator provided by the POWHEG-BOX based on MadGraph, or the FeynArts/FormCalc packages. However, the perturbatively meaningful and numerically stable evaluation of these contributions requires the design of a well-defined procedure for the treatment of single and double on-shell resonances that are associated with Born processes different from $p p \rightarrow \tilde{\chi}_{i} \tilde{\chi}_{j} j$, and should thus not be considered as real-emission corrections to that reaction. In particular, this includes the associated production of gluinos or squarks that subsequently decay into a weakino and a parton. Examples of diagrams that can become singly and or doubly resonant in specific regions of the SUSY parameter space are shown in figure $2(\mathrm{~b}, \mathrm{c})$ and figure $2(\mathrm{~d})$, respectively.

Note that single on-shell resonances emerge not only in weakino-pair plus jet production, but also in other supersymmetric production processes involving squarks or gluinos $[19,35-38]$. The necessity of the subtraction method we are referring to has first been described in ref. [35]. Technical details can also be found in ref. [36]. For the case of weakino-pair plus jet production the subtraction procedure of single resonances developed in ref. [19] can be used without significant changes. However, the subtraction of double resonances requires a more sophisticated scheme. The doubly resonant part of a matrix element for the production of the particle pairs $(i, j)$ and $(k, l)$ as sketched in figure 3 typically is of the form

$$
\mathcal{M}_{\text {res }}=A_{0} \frac{1}{\tilde{s}_{i j}} \times \frac{1}{\tilde{s}_{k l}}
$$




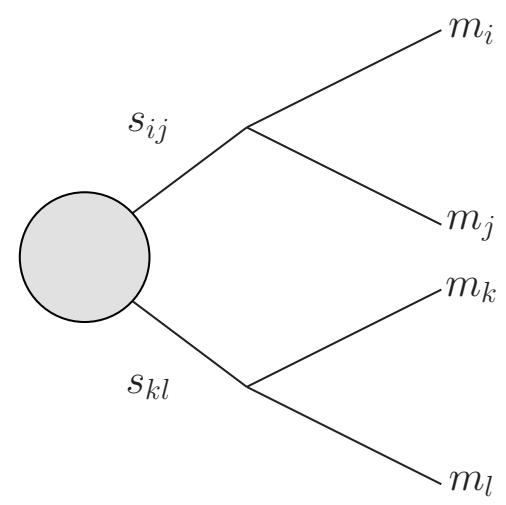

Figure 3. Final-state topology of a doubly resonant diagram for the production of the particle pairs $(i, j)$ and $(k, l)$.

where $A_{0}$ denotes the finite part of the amplitude, and the $1 / \tilde{s}_{a b}$ terms represent propagators that can go on-shell. For each pair of external particles $(a, b)$ we introduce the invariants $s_{a b}=\left(p_{a}+p_{b}\right)^{2}$ and $\tilde{s}_{a b} \equiv s_{a b}-m_{a b}^{2}$, where $m_{a b}$ is the mass of the resonant particle and $p_{a}, p_{b}$ are the four-momenta of the considered external particles. The amplitude $\mathcal{M}_{\text {res }}$ obviously diverges, if $\tilde{s}_{i j}$ and/or $\tilde{s}_{k l}$ approach zero.

Naively applying the regulator scheme we have used for the treatment of single resonances, by introducing a regulator $\Gamma_{\text {reg}}$, would result in a cross section that heavily depends on the regulator. Instead, we proceed by rewriting the resonant matrix element as

$$
\mathcal{M}_{\mathrm{res}}=\frac{A_{0}}{\tilde{s}_{i j}+\tilde{s}_{k l}} \times\left(\frac{1}{\tilde{s}_{i j}}+\frac{1}{\tilde{s}_{k l}}\right),
$$

effectively dividing the resonance structure into two single resonances, which can be treated separately. Since the resonance structure of the second factor appears already at Born level, we need to insert a regulator width only for the first term, using the same regulator for $(i, j)$ and $(k, l)$. The regulated matrix element now reads:

$$
\mathcal{M}_{\text {res }}=\frac{A_{0}}{\tilde{s}_{i j}+\tilde{s}_{k l}+i m_{i j} \Gamma_{\text {reg }}+i m_{k l} \Gamma_{\text {reg }}} \times\left(\frac{1}{\tilde{s}_{i j}}+\frac{1}{\tilde{s}_{k l}}\right) .
$$

In order to comply with the treatment of single resonances that already occur at Born level, additionally the physical decay widths $\Gamma_{i j}$ and $\Gamma_{k l}$ of the resonant particles have to be taken into account. The regulated matrix element can then be written as

$$
\mathcal{M}_{\text {res }} \equiv \mathcal{P}\left(\tilde{s}_{i j}, \tilde{s}_{k l}\right) A_{0},
$$

where $\mathcal{P}$ is the regulated propagator structure of the amplitude and is given by

$$
\begin{aligned}
\mathcal{P}\left(\tilde{s}_{i j}, \tilde{s}_{k l}\right)= & \frac{1}{\tilde{s}_{i j}+\tilde{s}_{k l}+i m_{i j}\left(\Gamma_{\mathrm{reg}}+\Gamma_{i j}\right)+i m_{k l}\left(\Gamma_{\mathrm{reg}}+\Gamma_{k l}\right)} \\
& \times\left(\frac{1}{\tilde{s}_{i j}+i m_{i j} \Gamma_{i j}}+\frac{1}{\tilde{s}_{k l}+i m_{k l} \Gamma_{k l}}\right) .
\end{aligned}
$$


Following the procedure of ref. [19], the calculation of a suitable counter-term for a resonant matrix element requires the determination of a Breit-Wigner factor that can be built by dividing the squared propagator structure itself by the squared propagator structure taken on-shell, where $\tilde{s}_{i j} \rightarrow 0, \tilde{s}_{k l} \rightarrow 0$ :

$$
\mathrm{BW}=\frac{\left|\mathcal{P}\left(\tilde{s}_{i j}, \tilde{s}_{k l}\right)\right|^{2}}{|\mathcal{P}(0,0)|^{2}} .
$$

Applying this procedure to the regulated doubly resonant matrix element leads to

$$
\begin{aligned}
\mathrm{BW}= & {\left[m_{i j}^{2} m_{k l}^{2} \Gamma_{i j}^{2} \Gamma_{k l}^{2}\left(m_{i j}\left(\Gamma_{i j}+\Gamma_{\mathrm{reg}}\right)+m_{k l}\left(\Gamma_{k l}+\Gamma_{\mathrm{reg}}\right)\right)^{2}\right.} \\
& \left.\times\left(\left(s_{i j}+s_{k l}-m_{i j}^{2}-m_{k l}^{2}\right)^{2}+\left(m_{i j} \Gamma_{i j}+m_{k l} \Gamma_{k l}\right)^{2}\right)\right] / \\
& {\left[\left(\left(s_{i j}-m_{i j}^{2}\right)^{2}+m_{i j}^{2} \Gamma_{i j}^{2}\right)\left(\left(s_{k l}-m_{k l}^{2}\right)^{2}+m_{k l}^{2} \Gamma_{k l}^{2}\right)\left(m_{i j} \Gamma_{i j}+m_{k l} \Gamma_{k l}\right)^{2}\right.} \\
& \left.\times\left(\left(s_{i j}+s_{k l}-m_{i j}^{2}-m_{k l}^{2}\right)^{2}+\left(m_{i j}\left(\Gamma_{i j}+\Gamma_{\text {reg }}\right)+m_{k l}\left(\Gamma_{k l}+\Gamma_{\text {reg }}\right)\right)^{2}\right)\right],
\end{aligned}
$$

which finally allows us to formulate the counter-term for doubly resonant matrix elements,

$$
\begin{aligned}
\left|\mathcal{M}_{\text {res }}^{\mathrm{CT}}\left(\Gamma_{\text {reg }}\right)\right|^{2}= & \Theta\left(\hat{s}-\left(m_{i j}+m_{k l}\right)^{2}\right) \Theta\left(m_{i j}-m_{i}-m_{j}\right) \Theta\left(m_{k l}-m_{k}-m_{l}\right) \\
& \times \mathrm{BW} \times\left|\mathcal{M}_{\text {res }}\left(\Gamma_{\text {reg }}\right)\right|_{\text {remapped }}^{2}
\end{aligned}
$$

Here $\hat{s}$ denotes the partonic center-of mass energy, and $m_{a}(a=(i j),(k l))$ the mass of an on-shell particle. The momenta entering $\mathcal{M}_{\text {res }}$ in the on-shell counter-term are to be remapped to the on-shell kinematics of the resonant particles, cf. ref. [36].

Similar to the singly resonant case, the cross section corresponding to the on-shell subtracted resonant terms in the doubly resonant case is obtained by subtracting the counter-term from the resonant matrix element and summing over all possible resonant channels:

$$
\sigma_{\text {real }}^{\text {OS }}=\sum_{\text {res }} \int d \Phi_{4}\left[\left|\mathcal{M}_{\text {res }}\left(\Gamma_{\text {reg }}\right)\right|^{2}-\mathcal{J}_{\text {res }}\left|\mathcal{M}_{\text {res }}^{\mathrm{CT}}\left(\Gamma_{\text {reg }}\right)\right|^{2}\right]
$$

where the Jacobian factor $\mathcal{J}_{\text {res }}$ reads

$$
\begin{aligned}
\mathcal{J}_{\text {res }}= & \frac{s_{i j} s_{k l}}{m_{i j}^{2} m_{k l}^{2}} \frac{\lambda^{1 / 2}\left(\hat{s}, m_{i j}^{2}, m_{k l}^{2}\right) \lambda^{1 / 2}\left(m_{i j}^{2}, m_{i}^{2}, m_{j}^{2}\right) \lambda^{1 / 2}\left(m_{k l}^{2}, m_{k}^{2}, m_{l}^{2}\right)}{\lambda^{1 / 2}\left(\hat{s}, s_{i j}, s_{k l}\right) \lambda^{1 / 2}\left(s_{i j}, m_{i}^{2}, m_{j}^{2}\right) \lambda^{1 / 2}\left(s_{k l}, m_{k}^{2}, m_{l}^{2}\right)} \\
& \times \frac{\left(\sqrt{\hat{s}}-m_{i j}\right)^{2}-\left(m_{k}+m_{l}\right)^{2}}{\left(\sqrt{\hat{s}}-\sqrt{s_{i j}}\right)^{2}-\left(m_{k}+m_{l}\right)^{2}} .
\end{aligned}
$$

Here, $\lambda$ denotes the usual Kaellen-function $\lambda(x, y, z)=x^{2}+y^{2}+z^{2}-2(x y+y z+z x)$, and $d \Phi_{4}$ is the full $2 \rightarrow 4$ phase-space element. For the actual evaluation of $\sigma_{\text {real }}^{\text {OS }}$ in the POWHEG-BOX, we have devised a routine allowing for a mapping of the phase-space according to a process-specific resonance structure. Because the real emission amplitudes require a special treatment for the removal of the doubly on-shell contributions, obtaining them with a default amplitude generator was not possible. We thus have used a modified 

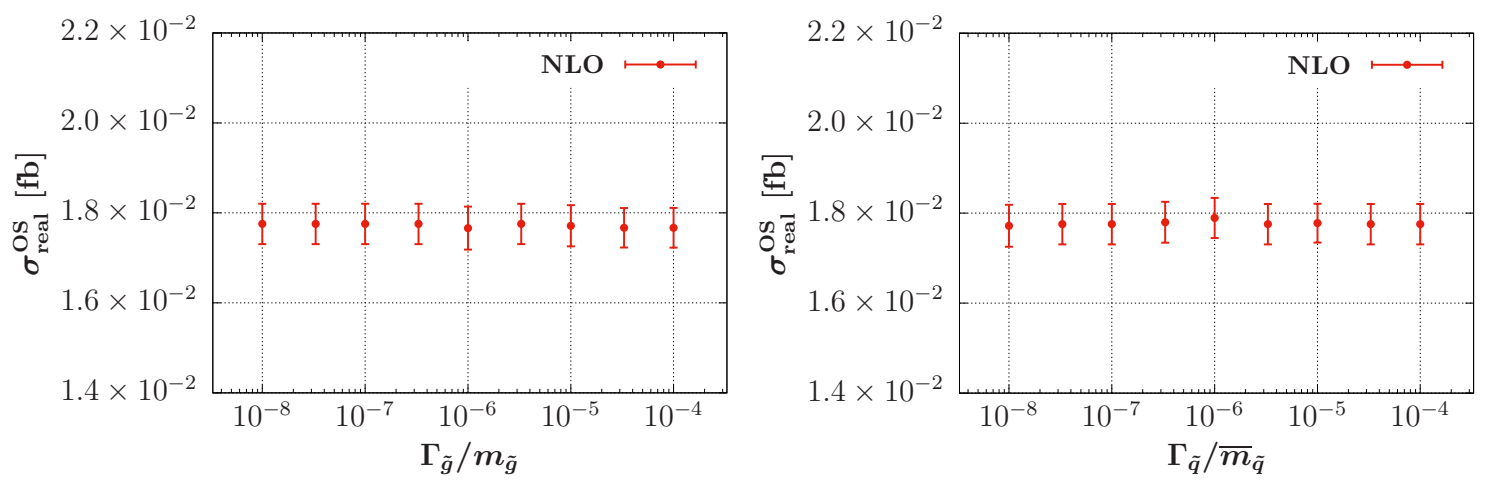

Figure 4. Dependence of the real on-shell contribution $\sigma_{\text {real }}^{\mathrm{OS}}$ to the cross section for the process $p p \rightarrow \tilde{\chi}_{1}^{0} \tilde{\chi}_{1}^{+} j$ with $\sqrt{s}=14 \mathrm{TeV}$ on the technical regulator $\Gamma_{\tilde{g}}$ (left) and $\Gamma_{\tilde{q}}$ (right).

version of FeynArts 3.9 [28] and FormCalc 9.4 [29] for the generation of the real-emission amplitudes and the identification of their resonance structure.

In order to verify the validity of our implementation, we have performed a number of checks. First, we have tested that, after the subtraction of on-shell resonances, for collinear momentum configurations real-emission and IR subtraction terms approach each other. Second, we have found that the dependence of our predictions for weakino-pair plus jet production cross sections on the technical regulator $\Gamma_{\text {reg }}$ is negligible. Figure 4 illustrates the regulator dependence of the on-shell resonant part of neutralino-pair production in association with a jet for a SUSY benchmark point that features squarks heavy enough to on-shell decay into a neutralino and a quark. Using the average of the four squark masses of the first generation, $\bar{m}_{\tilde{q}}=1.67 \mathrm{TeV}$, and the gluino mass $m_{\tilde{g}}=1.78 \mathrm{TeV}$, we find that in the range $\Gamma_{\tilde{q}} / \bar{m}_{\tilde{q}}=10^{-8}$ to $10^{-4}$ and $\Gamma_{\tilde{g}} / m_{\tilde{g}}=10^{-8}$ to $10^{-4}$, the dependence of the cross section on the regulator is entirely negligible, thus confirming the stability of the applied on-shell subtraction procedure. Additionally, we have checked that the proposed on-shell subtraction method preserves gauge invariance for the processes under consideration.

Finally, we have computed inclusive cross sections at LO and NLO accuracy for the pair production of the lightest neutralino in association with a jet in the setup of ref. [24] and found good agreement with the published results within the range of one percent. It is important to mention that the calculation described in ref. [24] does not include on-shell resonances, which we accounted for when comparing the results. Contributions of on-shell diagrams may be small for many SUSY parameter points and can be neglected in such cases, but they are essential for a consistent calculation in the most general case, as some allowed corners of the SUSY spectrum may lead to on-shell resonances.

\section{Phenomenological results}

A public release of the code for the production of a weakino pair with an additional identified jet will be made available in the framework of the POWHEG-BOX via the project website http://powhegbox.mib.infn.it. In this section we intend to highlight representative phenomenological results in order to demonstrate the capabilities of our code. 


\subsection{Choice of the spectrum and input parameters}

In order to calculate the physical SUSY spectrum and to obtain a SUSY Les Houches Accord (SLHA) file $[39,40]$ as input to our code, we chose a parameter point in the framework of the pMSSM10 [26] that is still allowed by current experimental limits on SUSY. The pMSSM10 is defined at the SUSY scale $M_{\mathrm{SUSY}}=\sqrt{m_{\tilde{t}_{1}} m_{\tilde{t}_{2}}}$, where $\tilde{t}_{1}$ and $\tilde{t}_{2}$ are the two stop mass-eigenstates, with ten soft SUSY breaking parameters, namely: the gaugino masses $M_{1}, M_{2}, M_{3}$, the first- and second-generation squark masses that are taken to be equal, $m_{\tilde{q}_{1}}=m_{\tilde{q}_{2}}$, the third-generation squark mass $m_{\tilde{q}_{3}}$, a common slepton mass for the three generations $m_{\tilde{\ell}}$, a common trilinear mixing parameter $A$ for the three generations, the Higgsino mass parameter $\mu$, the pseudo-scalar mass $M_{A}$, and $\tan \beta$, the ratio between the two vacuum expectation values of the Higgs fields. Left- and right-handed sfermion soft breaking masses are taken to be equal.

We have chosen a scenario in which the LSP is the lightest neutralino, with a reasonably low mass so that the production cross section is not too low. This has in particular lead to the following values of the ten parameters highlighted above,

$$
\begin{aligned}
& M_{1}=-120 \mathrm{GeV}, \quad M_{2}=160 \mathrm{GeV}, \quad M_{3}=1.70 \mathrm{TeV}, \\
& m_{\tilde{q}_{1}}=1.79 \mathrm{TeV}, \quad m_{\tilde{q}_{3}}=1.30 \mathrm{TeV}, \quad m_{\tilde{\ell}}=740 \mathrm{GeV}, \quad A=1.863 \mathrm{TeV} \text {, } \\
& \mu=190 \mathrm{GeV}, \quad M_{A}=1.35 \mathrm{TeV}, \quad \tan \beta=35 \text {. }
\end{aligned}
$$

We have used the SoftSUSY 4.0 program [41] for the calculation of the spectrum, and the SDECAY 1.3 program [42] for the calculation of the relevant decay widths and branching fractions to obtain the SLHA input file for our code in the POWHEG-BOX. Our electroweak input parameters are the $Z$ boson mass, $m_{Z}=91.1876 \mathrm{GeV}$, the electromagnetic coupling constant $\alpha^{-1}\left(M_{Z}\right)=127.934$, and the Fermi constant, $G_{F}=1.16637 \times 10^{-5} \mathrm{GeV}^{-2}$. The resulting neutralino masses are

$$
m_{\tilde{\chi}_{1}^{0}}=111.9 \mathrm{GeV}, \quad m_{\tilde{\chi}_{2}^{0}}=129.3 \mathrm{GeV}, \quad m_{\tilde{\chi}_{3}^{0}}=211.7 \mathrm{GeV}, \quad m_{\tilde{\chi}_{4}^{0}}=245.6 \mathrm{GeV},
$$

and the chargino masses are

$$
m_{\tilde{\chi}_{1}^{ \pm}}=130.9 \mathrm{GeV}, \quad m_{\tilde{\chi}_{2}^{ \pm}}=249.1 \mathrm{GeV} .
$$

The squark masses are equal for the first and second generation (and for up- and down-type squarks), but different for the third generation. They read

$$
\begin{aligned}
m_{\tilde{d}_{L} / \tilde{s}_{L}} & =m_{\tilde{u}_{L} / \tilde{c}_{L}}=1.836 \mathrm{TeV}, \\
m_{\tilde{b}_{1}} & =1.330 \mathrm{TeV}, \quad m_{\tilde{b}_{2}}=1.346 \mathrm{TeV}, \quad m_{\tilde{d}_{R} / \tilde{s}_{R}}=m_{\tilde{u}_{R} / \tilde{c}_{R}}=1.229 \mathrm{TeV}, \quad m_{\tilde{t}_{2}}=1.423 \mathrm{TeV} .
\end{aligned}
$$

\subsection{Cross sections and distributions at the LHC}

We consider proton-proton collisions at the LHC with a center-of-mass energy of $\sqrt{s}=$ $14 \mathrm{TeV}$. For the parton distribution functions (PDFs) of the proton we use for both the LO and NLO calculation the 'PDF4LHC15_nlo_nf4_30' PDF set [43] with four active flavors as implemented in the LHAPDF library [44] with ID $=92000$. We set the renormalization 

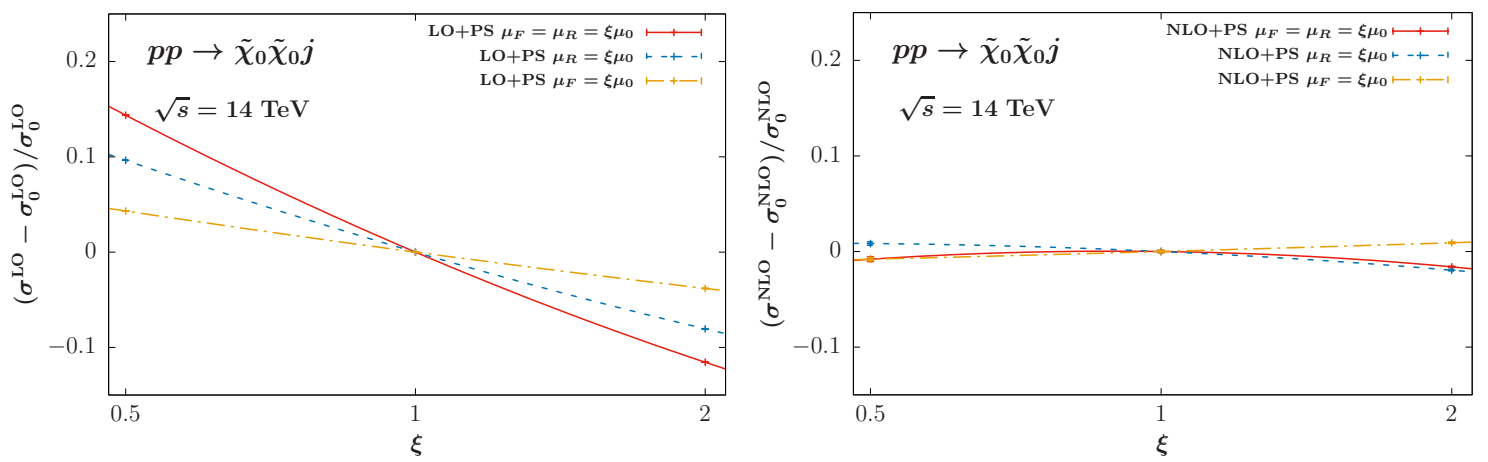

Figure 5. Dependence of the inclusive cross section for the process $p p \rightarrow \tilde{\chi}_{1}^{0} \tilde{\chi}_{1}^{0} j$ with $\sqrt{s}=14 \mathrm{TeV}$ within the cuts of eq. (3.5) on the factorization and renormalization scales. The curves show the deviation, $\left(\sigma-\sigma_{0}\right) / \sigma_{0}$, from the respective LO (left) or NLO (right) cross section, $\sigma_{0}=\sigma\left(\mu_{\mathrm{R}}=\right.$ $\left.\mu_{\mathrm{F}}=\mu_{0}\right)$, as a function of the scale parameter $\xi$, for three different cases: $\mu_{\mathrm{R}}=\mu_{\mathrm{F}}=\xi \mu_{0}$ (solid red line), $\mu_{\mathrm{R}}=\xi \mu_{0}, \mu_{\mathrm{F}}=\mu_{0}$ (dashed blue line), and $\mu_{\mathrm{R}}=\mu_{0}, \mu_{\mathrm{F}}=\xi \mu_{0}$ (dot-dashed yellow line). In each case, $\mu_{0}=2 m_{\tilde{\chi}_{1}^{0}}$.

and factorizations scales, $\mu_{\mathrm{R}}$ and $\mu_{\mathrm{F}}$, to be proportional to the sum of the masses of the weakinos $\tilde{\chi}_{A}$ and $\tilde{\chi}_{B}$ produced in the specific process under consideration, $\mu_{\mathrm{R}}=\mu_{\mathrm{F}}=\xi \mu_{0}$ with $\mu_{0}=m_{\tilde{\chi}_{A}}+m_{\tilde{\chi}_{B}}$, while the scale parameter $\xi$ is chosen to be one unless specifically stated otherwise. The fixed-order results are combined with the parton-shower program PYTHIA 6.4.25 [22] in which QED radiation, underlying event, and hadronization effects are switched off throughout. Partons arising from the real-emission contributions of the NLO-QCD calculation or from the parton shower are recombined into jets according to the anti- $k_{T}$ algorithm [45] as implemented in the FASTJET package [46] with $R=0.4$ and $\left|\eta^{\text {jet }}\right|<4.5$.

In the following we discuss phenomenological results for neutralino-pair production in association with a hard jet, $p p \rightarrow \tilde{\chi}_{1}^{0} \tilde{\chi}_{1}^{0} j$, in two different setups. For the first one only basic jet selection cuts are applied on the transverse momentum $p_{T}^{\text {jet }_{1}}$ and rapidity $\eta^{\text {jet }_{1}}$ of the hardest identified jet,

$$
p_{T}^{\text {jet }_{1}}>20 \mathrm{GeV}, \quad\left|\eta^{\text {jet }_{1}}\right|<4.5
$$

which results in a cross section of $1.37 \mathrm{fb}$ at NLO accuracy. The second set of cuts is inspired by experimental mono-jet searches [47-49]. Here, in addition to a severe cut on the hardest jet, an additional cut on the missing transverse momentum $p_{T}^{\text {miss }}$ is imposed,

$$
p_{T}^{\text {miss }}>100 \mathrm{GeV}, \quad p_{T}^{\text {jet }_{1}}>80 \mathrm{GeV}, \quad\left|\eta^{\text {jet }_{1}}\right|<2.8 .
$$

The missing transverse momentum is computed from the negative sum of detectable jets with a transverse momentum $p_{T}^{\text {jet }} \geq 30 \mathrm{GeV}$ and $\left|\eta^{\text {jet }}\right|<2.8$, similar to what is done in the experimental analyses. As the sum of the transverse momenta of the final-state particles needs to add to zero, this is effectively equivalent to the sum over the non-detected particles, more specifically the LSP and the softer jets.

Figure 5 illustrates the dependence of the LO and NLO cross sections within the cuts of eq. (3.5) on the scale parameter $\xi$. To quantify the theoretical uncertainties which emerge 

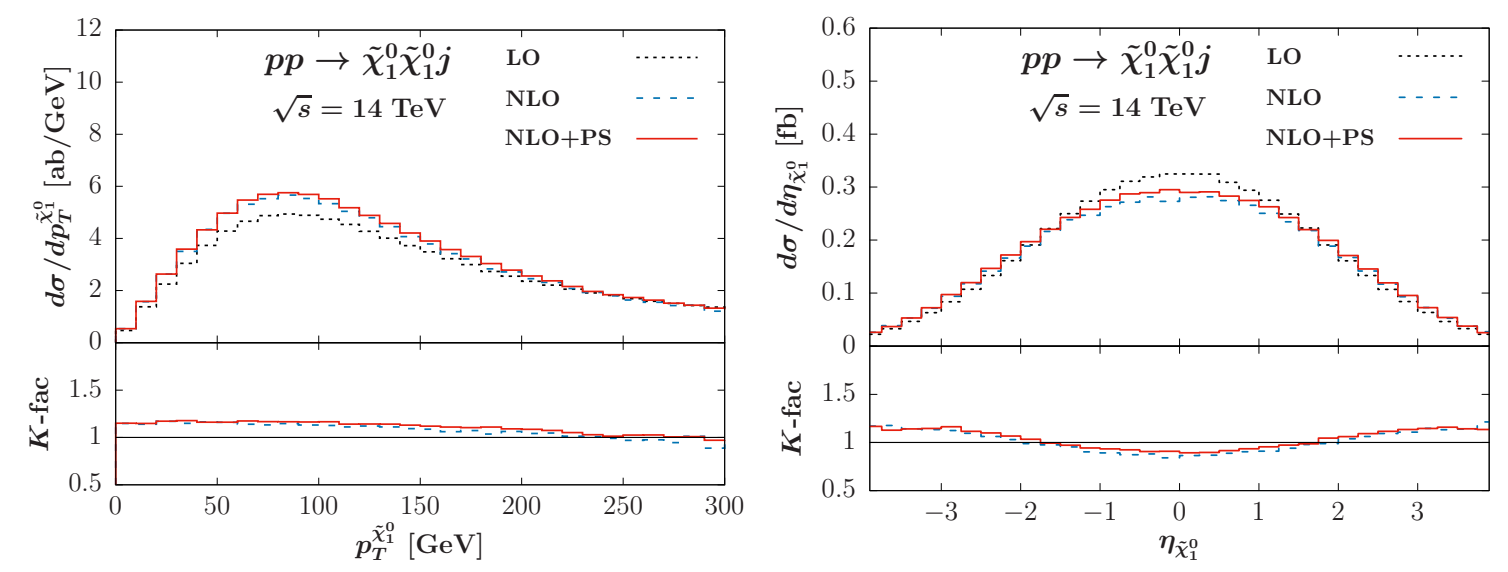

Figure 6. Average transverse-momentum $p_{T}^{\tilde{\chi}_{1}^{0}}$ (left) and pseudo-rapidity $\eta_{\tilde{\chi}_{1}^{0}}$ distributions (right) of the two neutralinos in the process $p p \rightarrow \tilde{\chi}_{1}^{0} \tilde{\chi}_{1}^{0} j$ at LO (dotted black lines), NLO (dashed blue lines), and NLO+PS (solid red lines) within the cuts of eq. (3.5) for our default setup.

from the unphysical renormalization and factorization scales, we have varied $\mu_{R}$ and $\mu_{F}$ in the range $0.5 \mu_{0}$ to $2 \mu_{0}$ around the default choice $\mu_{0} \equiv 2 m_{\tilde{\chi}_{1}^{0}}$. Since the LO cross section is already dependent on the strong coupling constant $\alpha_{s}$, neutralino-pair production in association with a jet depends not only on $\mu_{F}$ via the parton distribution functions of the scattering protons, but also on the renormalization scale $\mu_{R}$ entering the running of the strong coupling constant $\alpha_{s}$. At NLO, additional $\mu_{R}$ dependence occurs in the form of loop diagrams. However, in the considered range $0.5 \mu_{0}$ to $2 \mu_{0}$ the NLO cross section changes by only about $3 \%$, whereas the LO cross section changes by up to $14 \%$. This indicates that the perturbative expansion is stable, and the scale uncertainty at NLO is reduced remarkably compared to the LO calculation.

Characteristic distributions of the two neutralinos for the inclusive setup of eq. (3.5) are shown in figure 6 . In each case, the NLO prediction is significantly different from the respective LO curve. Adding the parton shower results in a small increase of the associated cross section. In the transverse-momentum distribution of the two neutralinos the NLO corrections are largest in the low- $p_{T}$ range, amounting to almost $15 \%$ while they decrease to less than $5 \%$ in the tail of the distribution. The parton shower increases the distribution uniformly over the entire range by about $5 \%$. In the average pseudo-rapidity distribution of the neutralinos, the corrections are negative in the central rapidity region, amounting to $-15 \%$. Towards larger values of $\eta_{\tilde{\chi}_{1}^{0}}$, the corrections are positive and increase the LO result by $15 \%$. Similar to the transverse-momentum distribution of the two neutralinos, the parton shower increases the $\eta_{\tilde{\chi}_{1}^{0}}$-distribution by $5 \%$ over the entire range. The transversemomentum distribution of the hardest jet, depicted in figure 7, exhibits a behavior slightly different than of the neutralinos. NLO corrections are small in the bulk, but increase up to $15 \%$ in the tail of the distribution. Parton-shower effects modify the fixed-order NLO results by an additional $5 \%$. Obviously, a constant $K$-factor would not account for these effects in each of these distributions. 

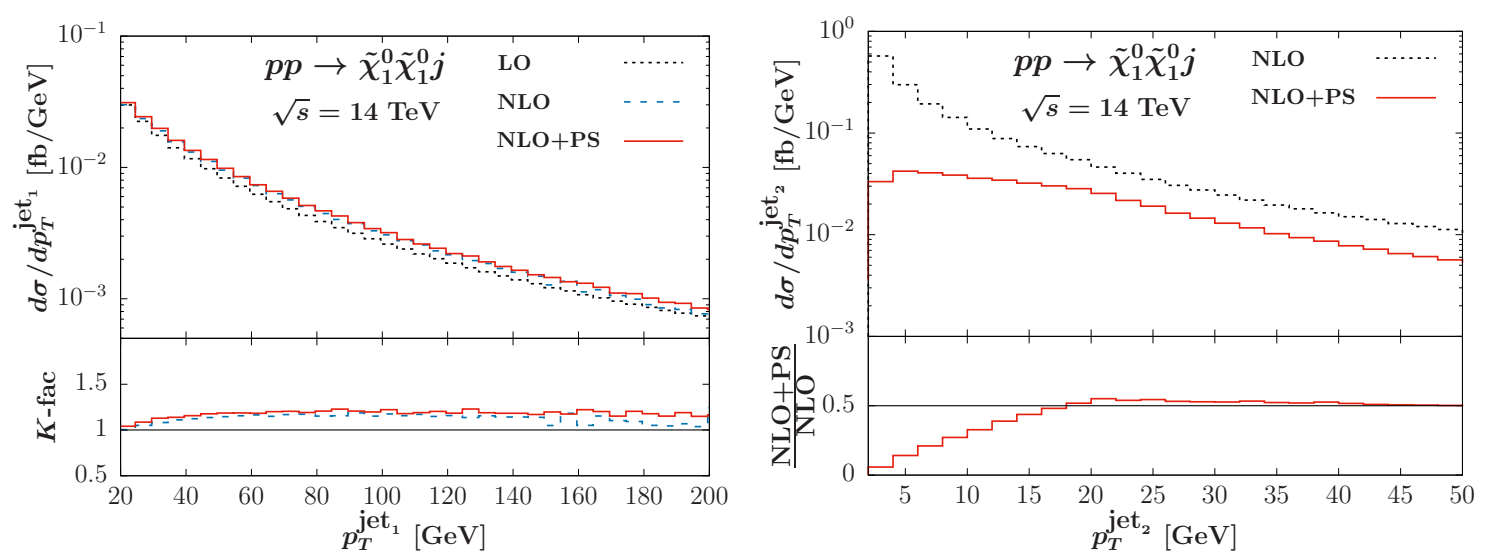

Figure 7. Transverse-momentum distribution of the hardest jet (left) and the second hardest jet (right) for the process $p p \rightarrow \tilde{\chi}_{1}^{0} \tilde{\chi}_{1}^{0} j$ at LO (dotted black lines), NLO (dashed blue lines), and NLO+PS (solid red lines) for our default setup, after the cuts of eq. (3.5) are applied.

While the distributions of the hardest jet can be described with full NLO accuracy by our calculation, the second-hardest jet is accounted for only by the real-emission contributions of the NLO-QCD corrections, and thus effectively only described with LO accuracy. Since no cuts are imposed on sub-leading jets in the setup we consider, it would be expected that the soft and collinear configurations dominate the behavior of such jets. This expectation is nicely confirmed for the transverse-momentum distribution of the second-hardest jet in figure 7 . The curve associated with the NLO calculation increases rapidly towards small values of $p_{T}^{\text {jet }_{2}}$ and exhibits a large negative entry in the lowest bin. The Sudakov factor of the NLO+PS implementation tames this increase.

Experimental searches for particles that cannot be directly identified in a default detector, such as neutralinos or, more generically, massive DM candidate particles, often rely on mono-jets. The tell-tale signature of such events consists in a hard jet that recoils off the system comprised by the heavy particles, accompanied by large missing transverse energy. To quantitatively account for such signatures an accurate description of the hard jet accompanying the heavy-particle system is of paramount importance. For the production of a neutralino pair in association with a hard jet that can give rise to a mono-jet signature we consider a scenario inspired by experimental searches with the cuts of eq. (3.6). Figure 8 illustrates the features of two characteristic distributions for such a scenario: the angular separation $\phi\left(\vec{p}_{\text {jet }_{1}}, \vec{p}_{\text {miss }}\right)$ of the hardest jet and of the missing momentum $\vec{p}_{\text {miss }}$; and the transverse component of the missing momentum, $p_{T}^{\text {miss }}$. At LO, the hard jet is produced back-to-back with the heavy neutralino system, resulting in an angular separation of $180^{\circ}$. At NLO, this back-to-back configuration can be altered by radiation effects due to real parton emission. Even more radiation occurs at the NLO+PS level, resulting in an additional smearing of the LO distribution and affecting in particular the last bin corresponding to $\phi=180^{\circ}$. The $p_{T}^{\text {miss }}$ distribution experiences a pronounced increase from LO to NLO and NLO+PS. While the NLO corrections are moderate in the bulk, they amount to more than $15 \%$ beyond about $150 \mathrm{GeV}$. A description of this behavior by a constant $\mathrm{K}$ factor would 

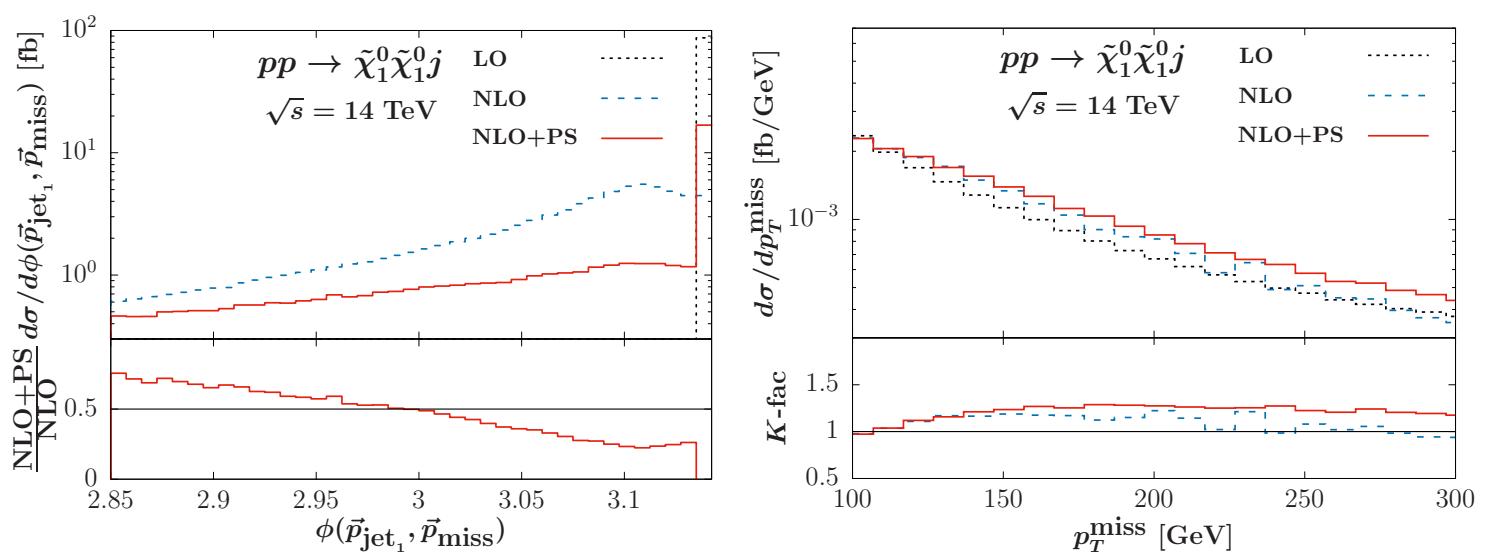

Figure 8. Angular separation $\phi$ between the missing momentum $\vec{p}_{\text {miss }}$ and the hardest jet momentum $\vec{p}_{\text {jet }}$ vectors (left) and missing transverse momentum $p_{T}^{\text {miss }}$ (right) for the process $p p \rightarrow \tilde{\chi}_{1}^{0} \tilde{\chi}_{1}^{0} j$ at LO (dotted black lines), NLO (dashed blue lines) and NLO+PS (solid red lines), after the cuts of eqs. (3.6) are applied.
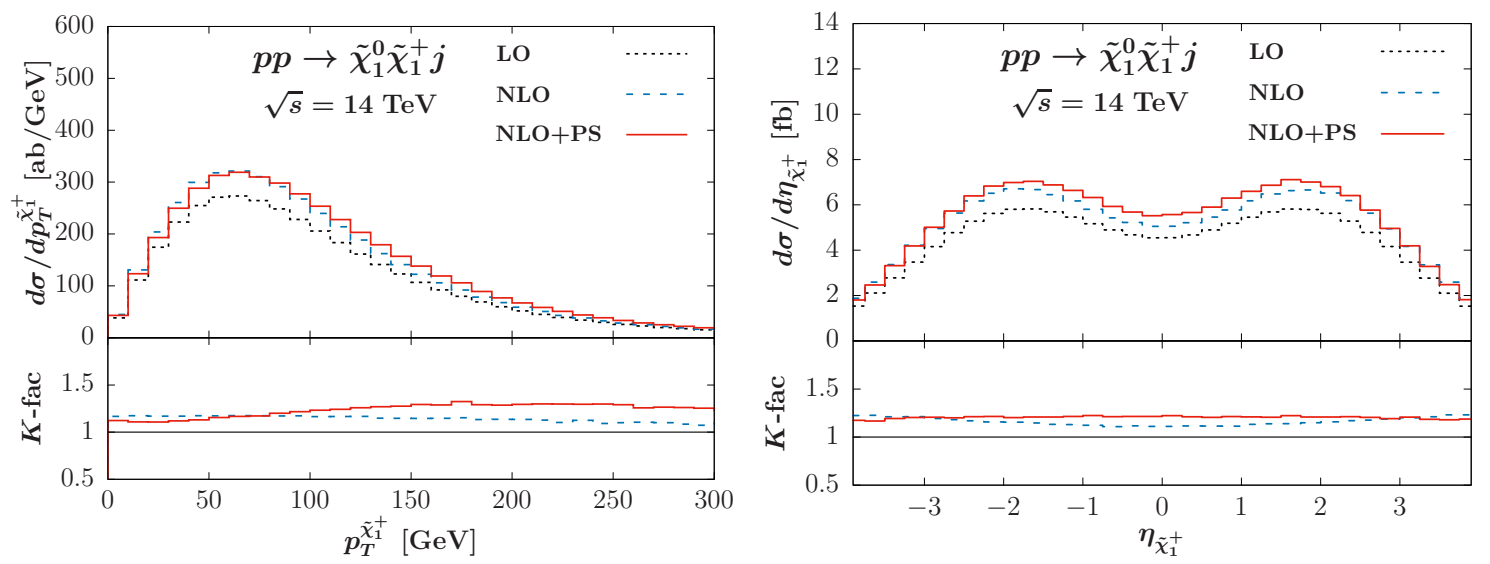

Figure 9. Transverse-momentum (left) and pseudo-rapidity distribution (right) of the chargino in the process $p p \rightarrow \tilde{\chi}_{1}^{0} \tilde{\chi}_{1}^{+} j$ at LO (dotted black lines), NLO (dashed blue lines), and NLO+PS (solid red lines) within the cuts of eq. (3.5) for our default setup.

clearly fail. The $p_{T}^{\text {miss }}$ distribution is increased uniformly by another $10 \%$ from NLO to NLO+PS by the parton-shower.

While a major motivation for the investigation of weakino-pair production processes at hadron colliders is the search for Dark Matter, this class of reactions is interesting also per se for the study of SUSY interactions. The code package we have developed thus not only allows for simulations of neutralino-pair production in association with a hard jet, but also for production processes involving various combinations of charginos and neutralinos. To demonstrate this feature of our work, we present in figure 9 representative distributions of the chargino produced in the reaction $p p \rightarrow \tilde{\chi}_{1}^{0} \tilde{\chi}_{1}^{+} j$ after the inclusive selection cuts of eq. (3.5) are applied. We find that the NLO (SUSY-)QCD corrections to the transversemomentum and pseudo-rapidity distributions of the chargino are similar in size to those for the related distributions in the case of neutralino-pair production (cf. figure 6). While 
we refrain from such a study in this work, we would like to point out that event files produced with our code in the default Les Houches format can easily be processed with public Monte-Carlo generators such as PYTHIA to simulate decays of the supersymmetric particles. In that case, not only distributions of the charginos, but also of their decay products can be simulated.

\section{Conclusions}

In this paper we have presented the implementation of weakino-pair production in association with an identified jet at a hadron collider in the framework of the POWHEG-BOX. The newly developed code allows for the calculation of the NLO SUSY-QCD corrections for the hard production process, and provides an interface to parton-shower programs such as PYTHIA via the POWHEG method. The program can process SLHA files obtained with an external spectrum calculator for the computation of a specific SUSY parameter point in the context of the MSSM. A generalized method to subtract on-shell resonances consistently in a $2 \rightarrow 3$ process has been formulated for the first time which could be helpful for many of other processes where the convergence of perturbation theory is spoiled by double on-shell resonances in the real corrections.

To illustrate the capabilities of the developed code package, we have discussed phenomenological features of a few selected processes focusing on theoretical uncertainties and the impact of parton shower effects on experimentally accessible observables. We have found that, in accordance with previous results reported in the literature, generally NLO corrections have a significant impact on production rates and reduce the scale uncertainty of the theoretical calculation. Parton-shower effects are small for weakino distributions, but are significant for jet observables, as expected. Thus, our work is of immediate relevance for mono-jet searches for Dark Matter at the LHC in the framework of the MSSM.

\section{Acknowledgments}

We are very grateful to Thomas Hahn for generous help with FormCalc and FeynArts. We also thank Marco Stratmann and Christoph Borschensky for valuable comments and discussions. We furthermore would like to thank the referee of our manuscript for feedback that helped to improve our article. This work has been supported in part by the Institutional Strategy of the University of Tübingen (DFG, ZUK 63), by the DFG Grant JA 1954/1, by the Carl Zeiss Foundation, and by the German Academic Scholarship Foundation (Studienstiftung des deutschen Volkes). J.B. also acknowledges the support in the last stages of this work from his Durham Senior Research Fellowship COFUNDed between Durham University and the European Union under grant agreement number 609412. This work was performed thanks to the support of the state of Baden-Württemberg through bwHPC and the German Research Foundation (DFG) through grant no INST 39/9631 FUGG. The Feynman diagrams of this paper have been drawn with the program JaxoDraw $2.0[50,51]$. 
Open Access. This article is distributed under the terms of the Creative Commons Attribution License (CC-BY 4.0), which permits any use, distribution and reproduction in any medium, provided the original author(s) and source are credited.

\section{References}

[1] ATLAS collaboration, Observation of a new particle in the search for the Standard Model Higgs boson with the ATLAS detector at the LHC, Phys. Lett. B 716 (2012) 1 [arXiv: 1207.7214] [INSPIRE].

[2] CMS collaboration, Observation of a new boson at a mass of $125 \mathrm{GeV}$ with the CMS experiment at the LHC, Phys. Lett. B 716 (2012) 30 [arXiv:1207.7235] [INSPIRE].

[3] D. Abercrombie et al., Dark Matter Benchmark Models for Early LHC Run-2 Searches: Report of the ATLAS/CMS Dark Matter Forum, arXiv:1507.00966 [INSPIRE].

[4] CMS collaboration, Searches for electroweak production of charginos, neutralinos and sleptons decaying to leptons and $W, Z$ and Higgs bosons in pp collisions at 8 TeV, Eur. Phys. J. C 74 (2014) 3036 [arXiv: 1405.7570] [INSPIRE].

[5] ATLAS collaboration, Search for direct pair production of a chargino and a neutralino decaying to the $125 \mathrm{GeV}$ Higgs boson in $\sqrt{s}=8 \mathrm{TeV}$ pp collisions with the ATLAS detector, Eur. Phys. J. C 75 (2015) 208 [arXiv:1501.07110] [INSPIRE].

[6] ATLAS collaboration, Search for the electroweak production of supersymmetric particles in $\sqrt{s}=8 \mathrm{TeV}$ pp collisions with the ATLAS detector, Phys. Rev. D 93 (2016) 052002 [arXiv: 1509.07152] [INSPIRE].

[7] CMS collaboration, Search for electroweak production of charginos in final states with two tau leptons in pp collisions at $\sqrt{s}=8 \mathrm{TeV}$, CMS-PAS-SUS-14-022 (2014).

[8] ATLAS collaboration, Search for the direct production of charginos and neutralinos in final states with tau leptons in $\sqrt{s}=13 \mathrm{TeV}$ pp collisions with the ATLAS detector, ATLAS-CONF-2017-035 (2017).

[9] ATLAS collaboration, Search for electroweak production of supersymmetric particles in the two and three lepton final state at $\sqrt{s}=13 \mathrm{TeV}$ with the ATLAS detector, ATLAS-CONF-2017-039 (2017).

[10] CMS collaboration, Combined search for electroweak production of charginos and neutralinos in pp collisions at $\sqrt{s}=13 \mathrm{TeV}$, CMS-PAS-SUS-17-004 (2017).

[11] W. Beenakker, M. Klasen, M. Krämer, T. Plehn, M. Spira and P.M. Zerwas, The production of charginos/neutralinos and sleptons at hadron colliders, Phys. Rev. Lett. 83 (1999) 3780 [Erratum ibid. 100 (2008) 029901] [hep-ph/9906298] [INSPIRE].

[12] W. Beenakker, R. Hopker and M. Spira, PROSPINO: A program for the production of supersymmetric particles in next-to-leading order QCD, hep-ph/9611232 [INSPIRE].

[13] J. Debove, B. Fuks and M. Klasen, Transverse-momentum resummation for gaugino-pair production at hadron colliders, Phys. Lett. B 688 (2010) 208 [arXiv:0907.1105] [InSPIRE].

[14] C.S. Li, Z. Li, R.J. Oakes and L.L. Yang, Threshold Resummation Effects in the Associated Production of Chargino and Neutralino at Hadron Colliders, Phys. Rev. D 77 (2008) 034010 [arXiv: 0707.3952] [INSPIRE].

[15] J. Debove, B. Fuks and M. Klasen, Threshold resummation for gaugino pair production at hadron colliders, Nucl. Phys. B 842 (2011) 51 [arXiv: 1005.2909] [INSPIRE]. 
[16] J. Debove, B. Fuks and M. Klasen, Joint Resummation for Gaugino Pair Production at Hadron Colliders, Nucl. Phys. B 849 (2011) 64 [arXiv:1102.4422] [inSPIRE].

[17] B. Fuks, M. Klasen, D.R. Lamprea and M. Rothering, Precision predictions for electroweak superpartner production at hadron colliders with Resummino, Eur. Phys. J. C $\mathbf{7 3}$ (2013) 2480 [arXiv: 1304.0790] [INSPIRE].

[18] H. Sun, L. Han, W.-G. Ma, R.-Y. Zhang, Y. Jiang and L. Guo, Full one-loop electroweak and NLO QCD corrections to the associated production of chargino and neutralino at hadron colliders, Phys. Rev. D 73 (2006) 055002 [hep-ph/0602089] [InSPIRE].

[19] J. Baglio, B. Jäger and M. Kesenheimer, Electroweakino pair production at the LHC: NLO SUSY-QCD corrections and parton-shower effects, JHEP 07 (2016) 083 [arXiv:1605.06509] [INSPIRE].

[20] P. Nason, A new method for combining NLO QCD with shower Monte Carlo algorithms, JHEP 11 (2004) 040 [hep-ph/0409146] [INSPIRE].

[21] S. Frixione, P. Nason and C. Oleari, Matching NLO QCD computations with Parton Shower simulations: the POWHEG method, JHEP 11 (2007) 070 [arXiv: 0709. 2092] [INSPIRE].

[22] T. Sjöstrand, S. Mrenna and P.Z. Skands, PYTHIA 6.4 Physics and Manual, JHEP 05 (2006) 026 [hep-ph/0603175] [INSPIRE].

[23] G.F. Giudice, T. Han, K. Wang and L.-T. Wang, Nearly Degenerate Gauginos and Dark Matter at the LHC, Phys. Rev. D 81 (2010) 115011 [arXiv:1004.4902] [InSPIRE].

[24] G. Cullen, N. Greiner and G. Heinrich, Susy-QCD corrections to neutralino pair production in association with a jet, Eur. Phys. J. C 73 (2013) 2388 [arXiv:1212.5154] [INSPIRE].

[25] S. Alioli, P. Nason, C. Oleari and E. Re, A general framework for implementing NLO calculations in shower Monte Carlo programs: the POWHEG BOX, JHEP 06 (2010) 043 [arXiv: 1002.2581] [INSPIRE].

[26] K.J. de Vries et al., The pMSSM10 after LHC Run 1, Eur. Phys. J. C 75 (2015) 422 [arXiv: 1504.03260] [INSPIRE].

[27] A. Denner and S. Dittmaier, The complex-mass scheme for perturbative calculations with unstable particles, Nucl. Phys. Proc. Suppl. 160 (2006) 22 [hep-ph/0605312] [InSPIRE].

[28] T. Hahn, Generating Feynman diagrams and amplitudes with FeynArts 3, Comput. Phys. Commun. 140 (2001) 418 [hep-ph/0012260] [INSPIRE].

[29] T. Hahn and M. Pérez-Victoria, Automatized one loop calculations in four-dimensions and D-dimensions, Comput. Phys. Commun. 118 (1999) 153 [hep-ph/9807565] [INSPIRE].

[30] T. Fritzsche, T. Hahn, S. Heinemeyer, F. von der Pahlen, H. Rzehak and C. Schappacher, The Implementation of the Renormalized Complex MSSM in FeynArts and FormCalc, Comput. Phys. Commun. 185 (2014) 1529 [arXiv:1309.1692] [INSPIRE].

[31] A. Denner, S. Dittmaier and L. Hofer, Collier: a fortran-based Complex One-Loop LIbrary in Extended Regularizations, Comput. Phys. Commun. 212 (2017) 220 [arXiv:1604.06792] [INSPIRE].

[32] S.P. Martin and M.T. Vaughn, Regularization dependence of running couplings in softly broken supersymmetry, Phys. Lett. B 318 (1993) 331 [hep-ph/9308222] [INSPIRE].

[33] W. Hollik and D. Stöckinger, Regularization and supersymmetry restoring counterterms in supersymmetric QCD, Eur. Phys. J. C 20 (2001) 105 [hep-ph/0103009] [INSPIRE].

[34] J.C. Collins, F. Wilczek and A. Zee, Low-Energy Manifestations of Heavy Particles: Application to the Neutral Current, Phys. Rev. D 18 (1978) 242 [INSPIRE]. 
[35] W. Beenakker, R. Hopker, M. Spira and P.M. Zerwas, Squark and gluino production at hadron colliders, Nucl. Phys. B 492 (1997) 51 [hep-ph/9610490] [INSPIRE].

[36] D. Gonçalves-Netto, D. López-Val, K. Mawatari, T. Plehn and I. Wigmore, Automated Squark and Gluino Production to Next-to-Leading Order, Phys. Rev. D 87 (2013) 014002 [arXiv: 1211.0286] [INSPIRE].

[37] R. Gavin et al., Matching Squark Pair Production at NLO with Parton Showers, JHEP 10 (2013) 187 [arXiv: 1305.4061] [INSPIRE].

[38] R. Gavin et al., Squark Production and Decay matched with Parton Showers at NLO, Eur. Phys. J. C 75 (2015) 29 [arXiv: 1407.7971] [inSPIRE].

[39] P.Z. Skands et al., SUSY Les Houches accord: Interfacing SUSY spectrum calculators, decay packages and event generators, JHEP 07 (2004) 036 [hep-ph/0311123] [INSPIRE].

[40] B.C. Allanach et al., SUSY Les Houches Accord 2, Comput. Phys. Commun. 180 (2009) 8 [arXiv: 0801.0045] [INSPIRE].

[41] B.C. Allanach, SOFTSUSY: a program for calculating supersymmetric spectra, Comput. Phys. Commun. 143 (2002) 305 [hep-ph/0104145] [INSPIRE].

[42] M. Mühlleitner, A. Djouadi and Y. Mambrini, SDECAY: A fortran code for the decays of the supersymmetric particles in the MSSM, Comput. Phys. Commun. 168 (2005) 46 [hep-ph/0311167] [INSPIRE].

[43] J. Butterworth et al., PDF 4LHC recommendations for LHC Run II, J. Phys. G 43 (2016) 023001 [arXiv: 1510.03865] [INSPIRE].

[44] A. Buckley et al., LHAPDF6: parton density access in the LHC precision era, Eur. Phys. J. C 75 (2015) 132 [arXiv: 1412.7420] [INSPIRE].

[45] M. Cacciari, G.P. Salam and G. Soyez, The anti-k(t) jet clustering algorithm, JHEP 04 (2008) 063 [arXiv:0802.1189] [INSPIRE].

[46] M. Cacciari, G.P. Salam and G. Soyez, FastJet User Manual, Eur. Phys. J. C 72 (2012) 1896 [arXiv:1111.6097] [INSPIRE].

[47] ATLAS collaboration, Search for the direct production of charginos and neutralinos in final states with tau leptons in $\sqrt{s}=13$ TeV pp collisions with the ATLAS detector, Eur. Phys. J. C 78 (2018) 154 [arXiv: 1708.07875] [inSPIRE].

[48] ATLAS collaboration, Search for new phenomena in final states with an energetic jet and large missing transverse momentum in pp collisions at $\sqrt{s}=8 \mathrm{TeV}$ with the ATLAS detector, Eur. Phys. J. C 75 (2015) 299 [arXiv:1502.01518] [InSPIRE].

[49] ATLAS collaboration, Monojet Analysis Performance Plots with $68-78 \mathrm{pb}^{-1}$ of $13 \mathrm{TeV}$ data collected by the ATLAS experiment, Tech. Rep. ATLAS-EXOT-2015-005, (2015).

[50] D. Binosi and L. Theussl, JaxoDraw: A graphical user interface for drawing Feynman diagrams, Comput. Phys. Commun. 161 (2004) 76 [hep-ph/0309015] [INSPIRE].

[51] D. Binosi, J. Collins, C. Kaufhold and L. Theussl, JaxoDraw: A graphical user interface for drawing Feynman diagrams. Version 2.0 release notes, Comput. Phys. Commun. 180 (2009) 1709 [arXiv: 0811.4113] [INSPIRE]. 